\title{
Assessing in silico the recruitment and functional spectrum of bacterial enzymes from secondary metabolism
}

\author{
Valery Veprinskiy ${ }^{2}$, Leonhard Heizinger ${ }^{1}$, Maximilian G. Plach ${ }^{1}$ and Rainer Merkl ${ }^{1 *}$ (D)
}

\begin{abstract}
Background: Microbes, plants, and fungi synthesize an enormous number of metabolites exhibiting rich chemical diversity. For a high-level classification, metabolism is subdivided into primary (PM) and secondary (SM) metabolism. SM products are often not essential for survival of the organism and it is generally assumed that SM enzymes stem from PM homologs.

Results: We wanted to assess evolutionary relationships and function of bona fide bacterial PM and SM enzymes. Thus, we analyzed the content of 1010 biosynthetic gene clusters (BGCs) from the MIBiG dataset; the encoded bacterial enzymes served as representatives of SM. The content of 15 bacterial genomes known not to harbor BGCs served as a representation of PM. Enzymes were categorized on their EC number and for these enzyme functions, frequencies were determined. The comparison of PM/SM frequencies indicates a certain preference for hydrolases (EC class 3) and ligases (EC class 6) in PM and of oxidoreductases (EC class 1) and lyases (EC class 4) in SM. Based on BLAST searches, we determined pairs of PM/SM homologs and their functional diversity. Oxidoreductases, transferases (EC class 2), lyases and isomerases (EC class 5) form a tightly interlinked network indicating that many protein folds can accommodate different functions in PM and SM. In contrast, the functional diversity of hydrolases and especially ligases is significantly limited in PM and SM.

For the most direct comparison of PM/SM homologs, we restricted for each BGC the search to the content of the genome it comes from. For each homologous hit, the contribution of the genomic neighborhood to metabolic pathways was summarized in BGC-specific html-pages that are interlinked with KEGG; this dataset can be downloaded from https://www.bioinf.ur.de.

Conclusions: Only few reaction chemistries are overrepresented in bacterial SM and at least 55\% of the enzymatic functions present in BGCs possess PM homologs. Many SM enzymes arose in PM and Nature utilized the evolvability of enzymes similarly to establish novel functions both in PM and SM. Future work aimed at the elucidation of evolutionary routes that have interconverted a PM enzyme into an SM homolog can profit from our BGC-specific annotations.
\end{abstract}

Keywords: Primary metabolism, Secondary metabolism, Enzyme evolution, Enzyme design

\section{Background}

Microbes synthesize a nearly astronomical number of metabolites that show rich chemical diversity and a broad range of biological activities. To achieve a highlevel classification, Kossel introduced already in 1891 the term "secondary" to distinguish less relevant metabolites from "essential" ones, which he named "primary" [1]. In

\footnotetext{
* Correspondence: Rainer.Merkl@ur.de

${ }^{1}$ Institute of Biophysics and Physical Biochemistry, University of Regensburg,

D-93040 Regensburg, Germany

Full list of author information is available at the end of the article
}

this way, he also coined the terms primary metabolism (PM) and secondary metabolism (SM). Since then, SM is defined as the sum of enzymatic reactions yielding natural compounds that are - in contrast to primary metabolites - not directly involved in growth, development or reproduction of an organism. Often, products of SM have an ecological function and serve as competitive weapons against other organisms, as agents of symbiosis or as sexual hormones [2]. Bacterial secondary metabolites are a rich source of antibiotics, chemotherapeutic drugs, and immune suppressants. Thus, they play 
important roles in medicine and are produced in large amounts by industrial microbiology. Nowadays, the complete genomic sequences of secondary metabolite producers are available and can be scanned rapidly to identify loci relevant for metabolite biosynthesis. Moreover, with recent genome editing and engineering techniques like CRISPR-Cas [3] allowing for rational pathway design, secondary metabolites are gaining new relevance [4].

Often, microbial secondary metabolites are derived by means of specific biosynthetic pathways and the corresponding genes are organized in biosynthetic gene clusters (BGCs) [5]. Compared to products of PM, secondary metabolites have a wider range of structures and biological activities [6]. This remarkable diversity reflects the random manner in which their biosynthesis has evolved. The pathways have been acquired opportunistically and horizontal gene transfer (HGT) of complete pathways concentrated in genomic islands is common [7]. However, horizontal gene transfer can only explain the propagation of already existing pathways but not the formation of the initial one; the latter process is unclear for most BGCs [8].

For the evolution of a novel SM pathway, it is generally assumed that it arises through the acquisition of genes from the PM repertoire $[6,9]$. According to this theory, after initial gene duplication of the PM predecessor, subsequent mutations shape the biological activity of the gene copy in a way that may give rise to "abnormal" products. If not directly beneficial, these products might become so after spontaneous chemical change or after modifications by other enzymes with broad substrate spectra, which may eventually result in a strain with a selective advantage [6]. Nowadays it is feasible to identify for an SM enzyme the primary precursor by means of computational biology and to confirm the most likely evolutionary route with the help of biochemical experiments. Thus, having chosen for a given SM enzyme the most likely PM predecessor, one can estimate the evolutionary cost needed for the genesis of a novel enzymatic function utilized in SM.

For example, chorismate is a central metabolic branch point molecule and the common precursor of primary (folate, tryptophan) and secondary metabolites (menaquinones, siderophores, antibiotics), which are vital for plants as well as free living and infectious microorganisms [10]. In PM, aminodeoxychorismate synthase (ADCS, folate biosynthesis) and anthranilate synthase (AS, tryptophan biosynthesis) form aminated chorismate derivatives. Both are heteromeric complexes consisting of the enzymes PabA/PabB or TrpG/TrpE, respectively. In SM, isochorismate synthase (ICS) hydroxylates chorismate for the synthesis of menaquinones and siderophores and is a homolog of PabB and TrpE [11]. We have recently reported on the biochemical conversion of an AS into an ICS by altering the nucleophile specificity of AS from ammonia to water. Interestingly, not more than two amino acid exchanges in a channel leading to the catalytic site were sufficient to interconvert AS into a bifunctional AS/ICS that can be utilized in SM [11].

The generally accepted hypothesis for BGC genesis, which assumes the recruitment of PM enzymes, is so far only based on the analysis of few enzyme families. For example, polyketide synthases (PKSs) and nonribosomal peptide synthases (NRPSs) have been traced back to their PM homologs $[7,8,12]$. However, a comprehensive compilation of such pairs of homologs it still missing. To fill this gap, we browsed the content of SM databases and identified homologous enzymes known to contribute to PM. We focused on metabolic enzymes from bacteria because their genomes are extensively annotated, which is a prerequisite for a detailed analysis. The enzyme pairs that we identified can be used now to elucidate modifications introduced by evolution in a PM enzyme to serve in SM and to guide conversion experiments similar to the one described above. Moreover, we characterized the evolvability of enzymes and the range of enzymatic functions occurring in bacterial SM and made plausible that for a minimum of 331 enzyme functions homologs occur both in PM and SM.

\section{Results}

\section{A compilation of bona fide PM and SM enzymes}

Unfortunately, the discrimination of PM and SM introduced by Kossel did not rely on function and was a purely phenomenological definition [8]. For example, lipids or polysaccharides are "essential" for every organism, but the synthesis of some of them is specific for a small class of species [9]. Therefore, it is often difficult to assign a metabolic pathway or an enzyme function exclusively to PM or SM. Databases like BRENDA [13] do not assign enzymes to PM or SM and the KEGG database [14] classifies too many enzymes as SM: For example, tryptophan biosynthesis belongs to PM in bacteria; however, the related gene products are annotated by KEGG as SM. It follows that one has to restrict the analysis to a carefully chosen subset, if one is interested to study enzymes, whose assignment to PM is without any doubt.

C. Hertweck and co-workers analyzed 211 complete genomes of anaerobic bacteria and identified 26 species that do not contain BGCs [15]. Among these 26 genomes lacking SM, we selected those that are integrated into KEGG, because we were dependent on a comprehensive annotation and we thus opted for the KEGG and BRENDA databases. In order to reduce phylogenetic bias, we eliminated closely related species; the names of the 15 remaining ones are listed in Table 1 . The annotations of these genomes were scanned to identify enzymes; the related 20370 sequences were added to the 
Table 1 Bacterial species known not to contain secondary metabolite gene clusters

\begin{tabular}{l}
\hline Bacterial species and description \\
\hline Dehalococcoides sp. VS \\
Chloroflexi (ph), Dehalococcoidia (cl), Dehalococcoid \\
Dehalococcoidaceae (fa), Dehalococcoides (gn) \\
Anaerobic, obligately organohalide-respiring \\
Dehalogenimonas lykanthroporepellens BL-DC-9 \\
Chloroflexi (ph), Dehalococcoidia (cl), Dehalogenimo \\
Strictly anaerobic, mesophilic, non spore-forming, \\
Gram-negative \\
Chloroflexus aurantiacus J-10-fl \\
Chloroflexi (ph), Chloroflexia (cl), Chloroflexales (or), \\
Chloroflexaceae (fa), Chloroflexus (gn) \\
Filamentous anoxygenic phototroph, thermophilic \\
green bacterium
\end{tabular}

Deferribacter desulfuricans SSM1

Deferribacteres (ph), Deferribacterales (or),

Deferribacteraceae (fa), Deferribacter (gn)

Strictly anaerobic, thermophilic, sulphur-reducing,

heterotroph

Calditerrivibrio nitroreducens Yu37-1

Deferribacteres (ph), Deferribacterales (or),

Deferribacteraceae $(\mathrm{fa})$

Strictly anaerobic, moderately thermophilic, nitrate-reducing,

Gram-negative, non-sporulating

Denitrovibrio acetiphilus N2460

Deferribacteres (ph), Deferribacterales (or), Deferribacteraceae

$(\mathrm{fa})$, Denitrovibrio (gn)

Obligately anaerobic, mesophilic, nitrate reducing

Flexistipes sinusarabici MAS10

Deferribacteres (ph), Deferribacterales (or), Deferribacteraceae

(fa), Flexistipes (gn)

Strictly anaerobic, moderately thermophilic, Gram-negative, non-motile, heterotrophic, marine habitat

Desulfurispirillum indicum $\mathrm{S} 5$

Chrysiogenetes (ph), Chrysiogenales (or), Chrysiogenaceae (fa), Desulfurispirillum (gn)

Strictly anaerobic, uses selenate, selenite, arsenate, nitrate or nitrite as terminal electron acceptors

Thermodesulfatator indicus CIR 29812

Thermodesulfobacteria (ph), Thermodesulfobacteriales (or), Thermodesulfobacteriaceae (fa), Thermodesulfatator (gn) Anaerobic, thermophilic, chemolithoautotrophic sulfate reducer isolated from a deep-sea hydrothermal vent

Thermanaerovibrio acidaminovorans Su883

Synergistetes (ph), Synergistia (cl), Synergistales (or), Synergistaceae (fa), Thermanaerovibrio (gn)

Anaerobic, isolated from an reactor of a sugar refinery,

Gram-negative, motile, non-spore-forming

Aminobacterium colombiense ALA-1

Synergistetes (ph), Synergistia (cl), Synergistales (or), Synergistaceae (fa), Aminobacterium (gn)

Isolated from an anaerobic lagoon, mesophilic, amino

acid fermenting, Gram-negative, non-sporulating

Thermovirga lienii Cas60314

Synergistetes (ph), Synergistia (cl), Synergistales (or),

Synergistaceae (fa), Thermovirga (gn)

Anaerobic, thermophilic, chemoorganotrophic,

Gram-negative, motile, from a marine oil well
Table 1 Bacterial species known not to contain secondary metabolite gene clusters (Continued)

Akkermansia muciniphila ATCC BAA-835

349741

Verrucomicrobia (ph), Verrucomicrobiae (cl),

Verrucomicrobiales (or), Akkermansiaceae (fa),

Akkermansia (gn)

Anaerobic, isolated from the human intestinal tract

552811 Thermus scotoductus SA-01

Deinococcus-Thermus (ph), Deinococci (cl), Thermales (or),

Thermaceae (fa), Thermus (gn)

Growth with oxygen and nitrate as terminal electron

acceptors, reduces a variety of metal ions

324602

Candidatus Cloacamonas acidaminovorans

Candidatus Cloacimonetes (ph), Candidatus Cloacimonas (gn)

Anaerobic digester of a municipal wastewater treatment plant

639282

The respective genomes are part of KEGG databases. The name, the NCBI

Tax-ID and the phylogenetic lineage are listed; abbreviations are: phylum (ph), class (cl), order (or), family (fa), genus (gn). Additionally, a short description of the habitat and of the species are given, which were taken from [19]

set enzymes $_{P M^{*}}$. Note that we use the label "PM", in order to explicitly indicate that we analyzed a specific and possibly uncomplete subset of enzyme functions contributing to PM.

Following the above arguments, we also had to restrict the analysis of SM enzymes by choosing well characterized cases. The most comprehensive compilation of microbial SM is the dataset Minimum Information about a Biosynthetic Gene cluster (MIBiG) [5]; each cluster has 717231 been individually annotated by experts in their fields. We analyzed 1010 bacterial BGCs of MIBiG (version 1) that contained 18390 proteins and identified 2724 enzymes with a precisely specified function. We named 653733 this set enzymes $S_{S M^{*}}$ to indicate that we selected a welldefined, but restricted subset of SM pathways.

\section{The enzymatic spectra of bacterial SM and PM overlap to a great extent}

The entries and annotations of the sets enzymes $_{P M^{*}}$ and enzymes $_{S M^{*}}$ were used to estimate enzymatic capabilities of PM and SM. To begin with, we determined for all entries the assigned EC numbers (EC\#) [16], because they specify unequivocally the catalyzed reactions. Moreover, EC numbers are organized in a hierarchical manner, which can be used to group similar reactions.

The first digit of each EC number is a class number $\left(E C \_c l\right)$ indicating one of six types of chemical reactions. The class EC 1 subsumes oxidoreductases that catalyze oxidation/reduction reactions and EC 2 transferases that transfer functional groups. EC 3 consists of hydrolases that catalyze the formation of two products from a substrate by hydrolysis and EC 4 contains lyases that catalyze the non-hydrolytic addition or removal of groups. The isomerases of EC 5 catalyze the intramolecular rearrangement within a single molecule and the ligases of EC 6 join together two molecules under consumption of ATP or similar triphosphates [17]. 
The second and third digits subdivide the reactions into subclasses $\left(E C \_s c\right)$ and subdivisions $\left(E C \_s d\right)$. The fourth digit is a serial number and addresses the substrate. Thus, if the first three digits of EC numbers are identical, the considered gene products belong to the same subdivision, $i$. e. share the same reaction chemistry. As we were interested to assess the occurrence of more general functions, we grouped enzymes on the first or up to the third EC digits, which is a common approach [18].

The 20370 entries of enzymes ${ }_{P M^{*}}$ have assigned 1197 different EC numbers. The normalized frequencies $f_{P M}$ " $\left(E C_{-} \#\right)$ were combined to assess the occurrence of more general reaction chemistries. Analogously, we determined normalized frequencies $f_{S M}\left(E C_{-} \#\right)$ and combined corresponding values. Table 2 lists these frequencies for EC classes, and Additional file 1: Table S1 those of subclasses, subdivisions, and of all EC numbers.

The $\mathrm{SM}^{*}$ enzymes have assigned 600 EC numbers. 331 of these enzyme functions occur both in $\mathrm{PM}^{*}$ and $\mathrm{SM}^{*}$ and 269 were exclusively found in enzymes eM* $^{*}$ On the other hand, enzymes ${ }_{P M^{*}}$ catalyze 866 specific functions not found in enzymes $M^{*}$. The enzyme functions of SM* belong to 123 different subdivisions. Not more than 13 of these subdivisions do not occur in enzymes ${ }_{P M^{*}}$ and ten of them are oxidoreductases. All $\mathrm{SM}^{*}$ frequencies can be found in Additional file 2: Table S2.

\section{Oxidoreductases and few other enzymes are key components of bacterial BGCs}

The comparison of the EC class frequencies listed in Table 2 shows that the classes EC 2 (transferases) and EC 5 (isomerases) are approximately equally abundant in enzymes $_{P M}$ and enzymes ${ }_{S M}$. The classes EC 3 (hydrolases) and EC 6 (ligases) are underrepresented to a certain degree and EC 1 (oxidoreductases) and EC 4 (lyases) are overrepresented in enzymes ${ }_{S M}$.

Why are oxidoreductases that catalyze oxidation/ reduction reactions, overrepresented in SM? Most of the species used to compile the set enzymes ${ }_{P M^{*}}$ live in

Table 2 Abundance of EC classes in enzymes ${ }_{P M^{*}}$ and enzymes ${ }_{S M^{*}}$

\begin{tabular}{lllllr}
\hline EC Class & Enzyme Function & $f_{P M^{*}}$ & $f_{S M^{*}}$ & $f_{\text {Ecoli }}$ & $f_{M y c o}$ \\
\hline 1 & Oxidoreductases & 18.71 & 22.10 & 19.86 & 8.38 \\
2 & Transferases & 35.25 & 36.33 & 33.57 & 41.32 \\
3 & Hydrolases & 17.25 & 11.64 & 25.13 & 22.16 \\
4 & Lyases & 10.70 & 17.28 & 9.45 & 2.99 \\
5 & Isomerases & 6.67 & 6.81 & 6.41 & 8.38 \\
6 & Ligases & 11.42 & 5.84 & 5.58 & 16.77 \\
\hline
\end{tabular}

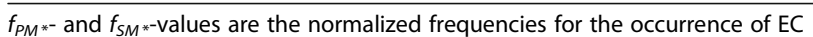
classes in the datasets enzymes $P_{P *}$ and enzymes ${ }_{S M^{*}} f_{E c o l i}$ and $f_{M y c o}$ are the frequencies of EC classes deduced from the genomes of $E$. coli and M. genitalium anaerobic habitats. As a consequence, the low frequency $f_{P M}$ ( $\left.E C_{-} c l\right)$ of oxidoreductases could be an artefact caused by a biased selection of PM enzymes in enzymes $_{P M *}$. In order to rule out a sampling bias and to further assess the effect of genome size on $f_{P M}\left(E C_{-} c l\right)$, we analyzed the Escherichia coli MG1655 genome (KEGG T00007) and that of Mycoplasma genitalium G37 (KEGG T00002). Both species are able to grow aerobically and anaerobically and $M$. genitalium is thought to have the smallest genome of any selfreplicating organism [19]. Although all abundancies vary noticeably, the frequency of encoded oxidoreductases is for both species smaller than in enzymes ${ }_{S M}$ *, which argues for a certain overrepresentation of oxidoreductases in bacterial SM and against a sampling bias in enzymes ${ }_{P M}$. This conclusion is in agreement with the known high SM abundance of oxygenases [20] and reflects that oxygen is a prerequisite for the synthesis of alkaloids and special antibiotics [21]. In a similar manner, lyases are more abundant in enzymes en* $^{*}$ than in enzymes $_{P M^{*}}$ and in the genomes of E. coli and M. genitalium; however, this bias is unclear to us.

For a more detailed analysis of functional spectra, we compared the frequencies of EC subdivisions ( $i$. $e$. reaction chemistries). Panel a of Fig. 1 is a plot of $f_{P M *}\left(E C \_s d\right)$ - versus $f_{S M *}\left(E C \_s d\right)$-values. Interestingly, the corresponding frequency pairs are moderately correlated with $r^{2}=0.51$, indicating that many functions occur in $\mathrm{PM}^{*}$ and $\mathrm{SM}^{*}$ with similar frequencies. Additionally, we determined the ratio overre$p\left(E C \_s d\right)=f_{S M}\left(E C \_s d\right) / f_{P M}\left(E C \_s d\right)$. Panel b of Fig. 1 shows that those subdivisions that are strongest overrepresented are also rare in enzymes eM* $^{*}$ and the overrepvalues indicate an even lower abundance in enzymes $P$ ** The most prominent subdivisions belong to EC 3.3.2 (ether hydrolases), EC 1.14.13 and EC 1.14.14 (oxidoreductases, acting on paired donors), EC 3.4.22 (cysteine endopeptidases), EC 4.3.99 (other carbon-nitrogen lyases), EC 5.3.3 (intramolecular oxidoreductases), and EC 5.4.4 (isomerases, transferring hydroxy groups). The corresponding enzyme functions are related to known key elements of SM, namely oxygen transfer, ether synthesis, or the nonribosomal biosynthesis of peptides [22]. On the other hand, the subdivisions that are most abundant in enzymes $_{S M^{*}}$ with $f_{S M^{*}}\left(E C \_s d\right)>0.05$, namely EC 4.2.1 (hydrolyases), EC 2.3.1 (acyltransferases, transferring groups other than amino-acyl groups), EC 1.1.1 (oxidoreductases, acting on the $\mathrm{CH}-\mathrm{OH}$ group of donors with $\mathrm{NAD}+$ or NADP+ as acceptor), and EC 2.7.7 (nucleotidyltransferases) occur in enzymes ${ }_{P M^{*}}$ with similar frequencies (overrep ()$\approx 1$ ). In summary, our findings suggest that the range of reaction chemistries used in $\mathrm{PM}^{*}$ and $\mathrm{SM}^{*}$ overlap to a great extent and that only few enzymes are highly specific for $\mathrm{SM}^{*}$. 

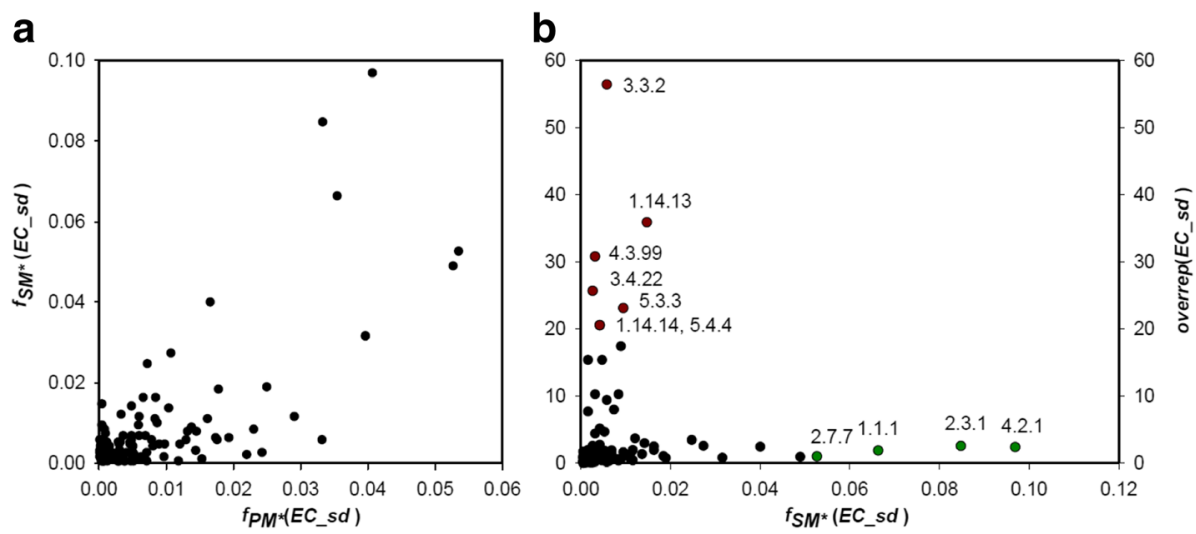

Fig. 1 Occurrence of EC subdivisions in $\mathrm{PM}^{*}$ and $S M^{*}$ and their overrepresentation in BGCs. a A plot of $f_{P M} *\left(E C \_s d\right)$-values versus $f_{S M *}\left(E C \_s d\right)$-values. These are the normalized frequencies for the occurrence of EC subdivisions in the datasets enzymes M $^{*}$ and enzymes SM, $^{*}$, respectively. $\mathbf{b}$ A plot of overrep $\left(E C_{-} s d\right)$-values versus $f_{S M} *\left(E C \_s d\right)$-values. Each overrep $\left(E C \_s d\right)$-value is the ratio $f_{S M} *\left(E C \_s d\right) / f_{P M} *\left(E C \_s d\right)$ that relates the abundance of a subdivision in enzymes $S_{M^{*}}$ and enzymes $S_{M^{*}}$. For subdivisions with an overrep()-value $>20$ (red symbols) and those most abundant in enzymes $S_{M^{*}}$ (green symbols), the EC_sd number is given

The distribution of monofunctional and multifunctional families is similar in enzymes ${ }_{P M^{*}}$ and enzymes ${ }_{S M^{*}}$

The above approach made plausible that approximately $55 \%$ of the enzymatic functions observed in enzymes ${ }_{S M}{ }^{*}$ are also present in enzymes ${ }_{P M}$. However, due to convergent evolution, enzymes that catalyze the same reaction do not necessarily possess the same 3D structure. We wanted to know whether these joint PM/SM enzyme functions have been established on the same or different protein folds.

Sequence alignments unambiguously distinguish proteins possessing similar and non-similar structures [23] and as stated by W.R. Pearson, homology of two protein sequences can be reliably inferred from a statistical significant BLAST hit [24]. In the following, we use homology as coined by W.R. Pearson as a term for similar structure ( $i$. e. identical fold) and common ancestry. Tracing the line of descent in more detail is difficult because BGCs and other SM functions are frequently acquired via horizontal gene transfer [7]. Thus, it is hard to decide whether the gene copies arose via speciation (orthologs) or gene duplication (paralogs) and whether the acquisition or gene genesis is a more recent or ancestral event.

We used blastp with the stringent cutoff $1 \mathrm{E}-20$ and searched in enzymes $_{P M^{*}}$ for hits related to enzymes from enzymes $_{S M *}$. These $\mathrm{PM}^{*} / \mathrm{SM}^{*}$ enzymes were considered as homologs. In order to eliminate false positive hits due to only one or few shared domains in multi-domain enzymes, we considered for each enzyme from enzymes sM $^{*}$ only those enzymes ${ }_{P M^{*}}$ hits that differed in length not more than $30 \%$ from the query.

For 269 enzyme functions from 27 subdivisions, we did not find a homolog in enzymes PM* $^{*} 81$ of the enzymes $_{S M} *$ only functions are oxidoreductases (EC class 1 ); this finding supports their $\mathrm{SM}^{*}$ overrepresentation determined above. However, our main goal was to characterize cases of SM* enzymes that can be traced back to PM* enzymes. Therefore, we concentrated on those SM* enzyme functions, for which BLAST found at least one $\mathrm{PM}^{*}$ hit. These were 331 enzyme functions from 96 subdivisions; thus we could significantly increase the set of SM enzymatic functions for which an origin in PM can be taken for granted.

These $\mathrm{PM}^{*} / \mathrm{SM}^{*}$ homologs are not necessarily isofunctional, because even a BLAST E-value below 1E-50 does not guarantee that the two compared sequences encode the same protein function [25]. For example, ICS (EC 5.4.4.2), PabB (EC 2.6.1.85), and TrpE (EC 4.1.3.27) are homologous [11] although their functions belong to three different EC classes. We were interested in assessing the rate of $\mathrm{SM}^{*}$ enzymes whose $\mathrm{PM}^{*}$ homologs catalyze different functions. This is why we compared for all EC subdivisions the EC numbers of enzymes ${ }_{S M^{*}}$ and their homologs in enzymes ${ }_{P M}$. For 48 cases, the $\mathrm{SM}^{*}$ enzymes and all of their $\mathrm{PM}^{*}$ homologs share the same subdivision. For 45 cases, $\mathrm{PM}^{*}$ homologs are from at least two different subdivisions, and for $3 \mathrm{SM}^{*}$ subdivisions, all $\mathrm{PM}^{*}$ homologs belong to a completely different subdivision. These findings indicate that approximately $39 \%(48 / 123)$ of these $\mathrm{SM}^{*}$ subdivisions are constituted by duplicated enzymes that utilize only one reaction chemistry both in enzymes ${ }_{P M^{*}}$ and enzymes en: $^{*}$ On the other hand, for $37 \%(45 / 123)$ of these $\mathrm{SM}^{*}$ subdivisions, their members belong to enzyme families that support in $\mathrm{PM}^{*}$ a larger spectrum of functions. The determined fraction of multifunctionality is a conservative approximation: When applying the cutoff $1 \mathrm{E}-10$, the number of monofunctional $\mathrm{SM}^{*} / \mathrm{PM}^{*}$ enzyme pairs (same EC subdivision) decreased to 42 , and that of multifunctional ones increased to 52 . 
However, lowering the stringency of this cutoff increases the risk of predicting false positives. As we were interested to identify highly reliable relationships, we utilized for the following analyses the conservatively chosen cutoff 1E-20.

To estimate in more detail the number of identical $\mathrm{PM}^{*} / \mathrm{SM}^{*}$ functions, we compared the full EC numbers. Of the $600 \mathrm{EC}$ numbers under study, the $\mathrm{SM}^{*}$ queries and their $\mathrm{PM}^{*}$ hits had the same number for 154 cases. Homologs with different EC numbers were found for 119 cases, and for 58 cases the EC number of all $\mathrm{PM}^{*}$ hits differed from the EC number of the $\mathrm{SM}^{*}$ query. Thus, of the 331 enzyme functions that occur both in enzymes $_{P M^{*}}$ and enzymes ${ }_{S M^{*}}, 46 \%$ belong to monofunctional families, $36 \%$ to multi-functional families and $18 \%$ most likely changed their function after recruitment from PM.

Are these three fractions to be expected? For a comparison, we BLASTed with the same parameters all enzymes enzymes genome $_{i}$ from each of the 15 genomes genome $_{i}$ constituting enzymes ${ }_{P M^{*}}$ against the specific set $\left\{\right.$ enzymes $_{P M *}$ enzymes $_{\text {genome }}$ \} that lacks the content of enzymes $_{\text {genome }_{i}}$, i. e. the $\mathrm{PM}^{*}$ enzymes found in one genome. $41 \%$ of the $\mathrm{PM}^{*} / \mathrm{PM}^{*}$ BLAST hits belonged to monofunctional enzyme families, $48 \%$ to multi-functional families and for $11 \%$ all hits had a different EC number. Analogously, the comparison of each set enzymes ${ }_{B G C_{i}}$ (enzymes from one BGC) against enzymes $_{S M *}$ enzymes $_{B G C_{i}}$ \} (content of all other BGCs) gave $41 \%$ monofunctional, $34 \%$ multi-functional enzyme families and $25 \%$ of the hits had a different EC number. The comparisons of the corresponding fraction values $\left(\mathrm{PM}^{*} / \mathrm{PM}^{*}\right.$ versus $\mathrm{PM}^{*} / \mathrm{SM}^{*}$ or $\left.\mathrm{SM}^{*} / \mathrm{SM}^{*}\right)$ indicate that the degree of neofunctionalization is similar in $\mathrm{PM}^{*}$ and $\mathrm{SM}^{*}$.

\section{Recruited SM enzymes reveal a typical pattern of functional flexibility}

It is known that a large portion (71\%) of all enzyme functions is performed by a relatively small set of 276 superfamilies [26]. Comparing the function of the corresponding members, it was shown that during enzyme evolution, $85 \%$ of functional changes led to enzymes belonging to the same EC class. The remaining $15 \%$ of the novel enzyme functions led to a change between EC classes. In $70 \%$ of these cases, enzymes from the EC classes 1, 2, and 3 were involved, and changes between isomerases and lyases (EC $4 \leftrightarrow E C 5$ ) were more frequent than expected [27].

We were interested to determine the functional flexibility of those enzymes that were recruited for SM and thus we related the EC numbers $E C_{-} \#_{-} S M^{*}$ occurring in enzymes $_{S M^{*}}$ and the EC numbers $E C_{-} \#_{-} P M^{*}$ of their homologous BLAST hits from enzymes ${ }_{P M^{*}}$. These abundancies were summarized on the level of EC classes and subdivisions, respectively, and for the corresponding BLAST E-values the mean was determined. Using Cytoscape [28], a network was created in which EC classes or subdivisions were represented by nodes and the number of PM hits and their mean E-values were used to determine width and color of interconnecting edges. These edges indicate a functional change $(\mathrm{PM} \rightarrow \mathrm{SM})$, because PM homologs possess a different function. Additionally, we determined the rate of functional conservation $f c\left(E C_{-} c l\right)$ by computing the fraction of $\mathrm{PM}^{*}$ homologs that belong to the same EC class or subdivision as the $\mathrm{SM}^{*}$ queries.

Figure 2 highlights three major trends on the class level: i) Oxidoreductases (EC class 1), transferases (EC class 2), lyases (EC class 4), and isomerases (EC class 5 ) form a tightly interlinked network indicating that many of these enzymes (i. e. folds) can adopt different functions in PM and SM. Among them, PM homologs of SM transferases support the widest functional spectrum indicated by the five edges ending in EC class 2 . The functional conservation $f c\left(E C_{-} c l\right)$ was below 0.9 only for lyases $(f c=0.74)$ and for isomerases $(f c=0.60)$ indicating

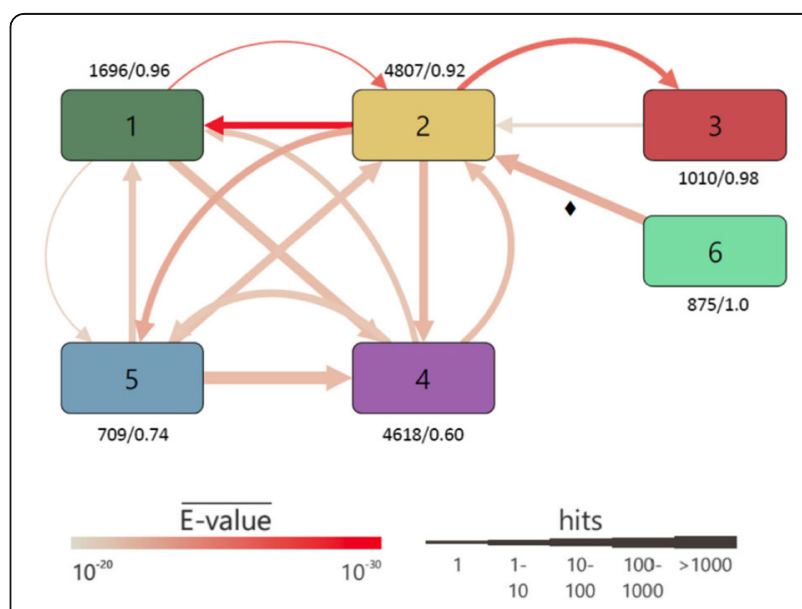

Fig. 2 Multifunctionality deduced from homologous $\mathrm{PM}^{*} / \mathrm{SM}^{*}$ pairs and determined for EC classes. The nodes represent the six EC classes and arrows indicate the relation of functional difference $\mathrm{PM}^{*} \rightarrow \mathrm{SM}^{*}$. For example, the arrow $6 \rightarrow 2$ signals that $\mathrm{PM}^{*}$ homologs of $\mathrm{SM}^{*}$ class 2 enzymes belong to EC class 6; this arrow is marked with a symbol. The width of the arrows represents the number of BLAST hits of enzymes from enzymes ${ }_{M^{*}}$ in enzymes ${ }_{P M^{*}}$ and their color the mean E-value; hits were binned as indicated. In addition, for each class, the number of PM* BLAST hits is given and the rate of functional conservation $f c$, which is the fraction of $\mathrm{PM}^{*}$ BLAST hits that belong to the same EC class as the $\mathrm{SM}^{*}$ queries. The class EC 1 subsumes oxidoreductases that catalyze oxidation/reduction reactions and EC 2 transferases that transfer functional groups. EC 3 consists of hydrolases that catalyze the formation of two products from a substrate by hydrolysis and EC 4 contains lyases that catalyze the non-hydrolytic addition or removal of groups. The isomerases of EC 5 catalyze the intramolecular rearrangement within a single molecule and the ligases of EC 6 join together two molecules under consumption of ATP or similar triphosphates 
that a large fraction of the homologs catalyze completely different reactions in $\mathrm{PM}^{*}$. ii) In contrast, for $\mathrm{SM}^{*}$ hydrolases (EC class 3) the functional flexibility of $\mathrm{PM}^{*}$ homologs is limited to transferases (EC class 2). Thus, although hydrolases are abundant in enzymes ${ }_{P M^{*}}$ and enzymes $_{S M^{*}}$ (Table 2), few are from multifunctional families, which may be due to their special chemistry of cleaving bonds by adding $\mathrm{H}_{2} \mathrm{O}$. iii) None of the $\mathrm{PM}^{*}$ homologs of $\mathrm{SM}^{*}$ ligases (EC class 6) had a function belonging to a different EC class. Ligases catalyze the joining of two molecules by hydrolyzing ATP or other triphosphates. It seems difficult to integrate this functionality into scaffolds from EC class $1-5$ enzymes. The limited flexibility of EC class 3 and EC class 6 enzymes is not an artefact caused by a too stringent cutoff. We lowered the BLAST cutoff to $1 \mathrm{E}-10$ and repeated the analysis of functional flexibility. The resulting graph is shown in Additional file 3: Figure S1. It contains only one additional arrow $(6 \rightarrow 2)$, which is compatible with the above findings.
For a more detailed analysis, we computed an analogous network (cutoff 1E-20) on the level of subdivisions $\left(E C \_s d\right)$, which is shown in Fig. 3. For each SM* subdivision $E C \_s d \_S M^{*}$, all subdivisions were determined that contained $\mathrm{PM}^{*}$ homologs. Thus, each directed edge $\left(E C \_s d \_P M^{*} \rightarrow E C \_s d \_S M^{*}\right)$ of the network signals an additional reaction chemistry found in some of the $\mathrm{PM}^{*}$ homologs. The graph contains six isolated edges and a 2-edge subgraph proposing the limited functional diversity of the corresponding PM homologs. Interestingly, two larger networks arose that subsume enzymes from the EC classes 1, 2, 4, and 5, and from the EC classes 1, 2,4 , and 6 , respectively. In the following, we concentrate on the most versatile subdivisions being interconnected in Fig. 3 by reddish and wide arrows. Among oxidoreductases, those that act on the $\mathrm{CH}-\mathrm{OH}$ group of donors with $\mathrm{NAD}(+)$ or $\mathrm{NADP}(+)$ as acceptor (EC 1.1.1) and among transferases, transaminases (EC 2.6.1) possess high evolvability. The high functional flexibility of lyases is due to the evolvability of carboxy-lyases (EC 4.1.1),
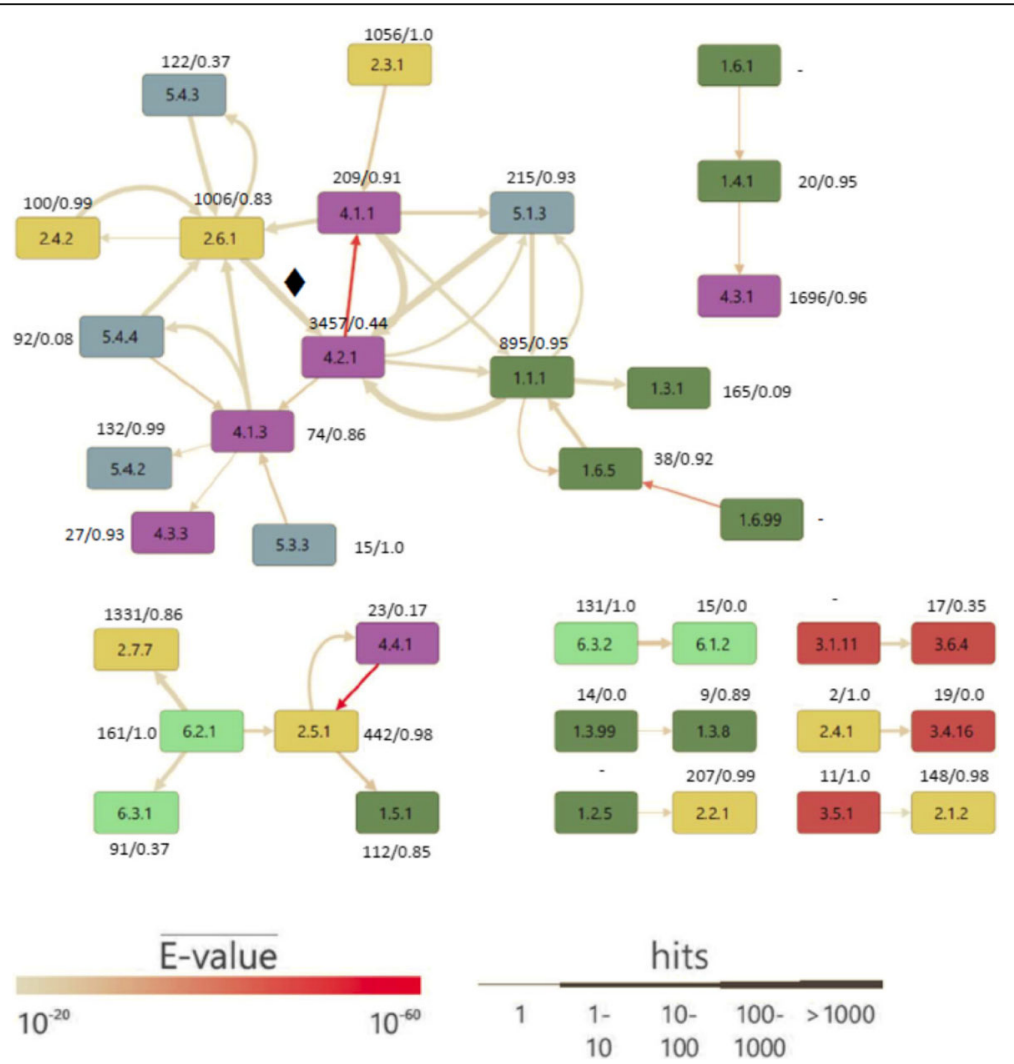

Fig. 3 Multifunctionality deduced from homologous PM/SM pairs and determined for EC subdivisions. The nodes represent EC subdivisions and arrows indicate the relation of functional difference $\mathrm{PM}^{*} \rightarrow \mathrm{SM}^{*}$. For example, the arrow 2.6.1 $\rightarrow$ 4.2.1 signals that $\mathrm{PM}^{*}$ homologs of $\mathrm{SM}^{*}$ subdivision 4.2.1 belong to EC subdivision 2.6.1; this arrow is marked with a . The width of the arrows represents the number of BLAST hits of enzymes from enzymes $S_{M^{*}}$ in enzymes PM $^{*}$ and their color the mean E-value; hits were binned as indicated. In addition, for each subdivision, the number of PM* BLAST hits is given and the rate of functional conservation $f c$, which is the fraction of PM* BLAST hits that belong to the same EC subdivision as the $\mathrm{SM}^{*}$ queries. Subdivisions that do not occur in enzymes ${ }_{P M^{*}}$ are indicated by a "-" 
oxo-acid-lyases (EC 4.1.3) and hydro-lyases (EC 4.2.1) and among isomerases, this holds for racemases and epimerases acting on carbohydrates (EC 5.1.3). The lowest functional conservation $f c\left(E C \_s d\right)$ of the subdivisions with more than $50 \mathrm{PM}^{*}$ hits possess oxidoreductases acting on $\mathrm{CH}-\mathrm{CH}$ groups (EC 1.3.1, $f_{c}=0.09$ ), hydrolyases (EC 4.2.1, $f c=0.44$ ), isomerases transferring amino groups (EC 5.4.3, $f c=0.37$ ), isomerases transferring hydroxy groups (EC 5.4.4, $f c=0.08$ ), and amide synthases (EC 6.3.1, $f c=0.37$ ). In summary, Fig. 3 confirms that the most drastic changes of reaction chemistry associated with the recruitment for $\mathrm{SM}^{*}$ occur in isomerases and lyases, which are known as functionally flexible [27].

One can understand many of these multifunctionalities by comparing the substrates and the chemistry of the enzymes. For example, the common substrate of the oxo-acid lyase TrpE (EC 4.1.3.27) and the intermolecular transferase ICS (EC 5.4.4.2) is chorismate and the interconversion of these two enzymatic functions has been demonstrated recently [11]. An other example are the $\mathrm{SM}^{*}$ enzyme 2,3-dihydroxybenzoate-AMP ligase (EC 2.7.7.58) that transfers 2,3-dihydroxybenzoate onto ATP and the $\mathrm{PM}^{*}$ enzyme o-succinylbenzoyl-coenzyme A synthetase (EC 6.2.1.26) that transfers 2-succinylbenzoate onto ATP. Thus, in both reactions a carboxylic acid substrate is transferred to ATP to give an acid-adenylate.

\section{A compilation of genomic neighborhoods that support the detailed characterization of homologous PM/SM enzyme pairs}

For a direct comparison, those pairs of homologous enzymes are of great interest that are located in the genome of one species and contribute to PM or SM, respectively. In order to make possible a detailed analysis for the user, we restricted the analysis to those 339 BGCs that are annotated in KEGG and named this set $B G C_{K E G G}$. These BGCs contain 4856 gene products; according to their GO terms [29] 3156 are enzymes; 937 have assigned one of 396 different EC numbers. Based on KEGG annotations and GO terms, we identified all enzymes enzymes species $C_{B E G G}$ from a single $B G C_{K E G G}$. Then, the full genome, $i$. e. the DNA sequence of the respective species was scanned for homologs of each set enzymes species $_{B \in G G G}^{\text {spy }}$ by using tblastn with a cutoff of $1 \mathrm{E}-20$ and all BLAST hits were added to a BGC-specific html-page.

Each enzyme from enzymes species $_{B G G G}$ may possess - in the same genome - several homologs and it is difficult to decide for each BLAST hit put $_{P M}$ whether it is part of PM or SM, since the functional annotation of a single enzyme may be misleading. As a first additional clue, the label "P" (indicating a possible contribution to PM) was assigned to each gene product, if at least one element of enzymes $_{P M^{*}}$ had the same EC number. The label "S" (indicating a possible contribution to $\mathrm{SM}$ ) was assigned, if KEGG mapped this enzyme function to the speciesspecific pathway "Biosynthesis of secondary metabolites". Moreover, the genomic neighborhood of a $p t_{P M}$ may assist the user with classification, because in bacteria, more than $50 \%$ of the genes are organized in operons and the gene products are often involved in the same functional pathway [30]. Thus, each $p u t_{P M^{-}}$-specific \pm 10 gene neighborhood was added to the html-pages as an additional block of information. These neighbors were further annotated by means of KEGG data and for each gene, a link to the respective KEGG GENES database entry was implemented, which allows for a rapid access to the comprehensive annotation deposited there. To provide further support for the contribution of put $_{P M}$ to metabolic pathways, the KEGG PATHWAY annotation of the \pm 10 and the \pm 2 gene neighborhood of each $p u t_{P M}$ were summarized. These two numbers were chosen, because the average operon length deduced for 42 bacterial species is three to four genes and in the genome of the typical bacterium E. coli, more than $95 \%$ of all operons are shorter than ten genes [31]. Taken together, a genomic neighborhood annotated predominantly with "P" encodes most likely a PM pathway and one can further corroborate this hypothesis by assessing the corresponding KEGG PATHWAY annotations. Combining these data, one can identify such candidates put $_{P M}$, whose PM membership is highly plausible.

To illustrate the usefulness of these annotations, we detail four cases. Table 3 represents part of the htmlpage related to BGC000309. This MIBiG cluster specifies the bacillibactin biosynthetic gene cluster (SM) from $\mathrm{Ba}$ cillus subtilis. It contains the gene bsu:BSU_31990, whose product is annotated as an isochorismate synthase (EC 5.4.4.2). One B. subtilis homolog with an E-value of 5E-26 is bsu:BSU00740; the gene product is annotated as PabB (EC 2.6.1.85) and is a subunit of the heterodimeric para-aminobenzoate synthase involved in folate biosynthesis (PM). 13 gene products encoded within the corresponding \pm 10 gene neighborhood of $p a b \mathrm{~B}$ have assigned a "P" and not more than two an "S". Six genes of the \pm 10 and 3 of the \pm 2 neighborhood are involved in folate biosynthesis. In summary, these annotations make clear that this DhbC homolog, named PabB, is a PM enzyme.

As explained in the Introduction, the ICS DhbC possesses a further PM homolog, which is an anthranilate synthase (EC 4.1.3.27) [11]. As expected, our analysis identified this SM/PM enzyme pair as well: A second DhbC homolog with an E-value of 3E-20 is bsu:BSU22680 and its neighborhood is depicted in Table 4. This gene product is one subunit of the heterodimeric anthranilate synthase involved in tryptophan biosynthesis. However, 
Table 3 Annotation of the \pm 10 genomic neighborhood of pabB from B. subtilis

\begin{tabular}{|c|c|c|c|}
\hline E_PM & K_SM & KEGG Annotation & Pathways in $\pm 10 / \pm 2 \mathrm{Nh}$ \\
\hline $\mathrm{P}$ & & $\begin{array}{l}\text { bsu:BSU00640 spoIIE; stage II sporulation protein E (EC } \\
3.1 .3 .16 \text { ) } \\
\text { bsu:BSU00650 yabS; hypothetical protein; K07114 Ca-activated } \\
\text { chloride channel homolog }\end{array}$ & \multirow{19}{*}{$\begin{array}{l}6 / 3 \text { bsu00790 Folate biosynthesis } \\
2 / 1 \text { bsu01110 Biosynthesis of } \\
\text { secondary metabolites } \\
1 / 1 \text { bsu01130 Biosynthesis of } \\
\text { antibiotics } \\
1 / 1 \text { bsu00270 Cysteine and } \\
\text { methionine metabolism } \\
1 / 1 \text { bsu00920 Sulfur metabolism } \\
1 / 0 \text { bsu00970 Aminoacyl-tRNA } \\
\text { biosynthesis } \\
1 / 0 \text { bsu01200 Carbon metabolism } \\
1 / 0 \text { bsu00770 Pantothenate and } \\
\text { CoA biosynthesis } \\
1 / 0 \text { bsu00230 Purine metabolism } \\
1 / 1 \text { bsu01230 Biosynthesis of } \\
\text { amino acids }\end{array}$} \\
\hline $\mathrm{P}$ & & $\begin{array}{l}\text { bsu:BSU00660 уаbT; serine/threonine protein kinase (EC } \\
2.7 .11 .1 \text { ) }\end{array}$ & \\
\hline $\mathrm{P}$ & & $\begin{array}{l}\text { bsu: BSU00670 tils; tRNA(ile)-lysidine synthase; K04075 } \\
\text { tRNA(Ile)-lysidine synthase [EC 6.3.4.19] }\end{array}$ & \\
\hline $\mathrm{P}$ & $\mathrm{S}$ & $\begin{array}{l}\text { bsu:BSU00680 hprT; hypoxanthine-guanine phosphoribosyltrans- } \\
\text { ferase (EC } 2.4 .2 .8 \text { ) } \\
\text { bsu:BSU00690 ftsH; ATP-dependent zinc metalloprotease FtsH (EC } \\
3.4 .24\end{array}$ & \\
\hline \multirow[t]{3}{*}{$P$} & & bsu:BSU00700 coaX; type III pantothenate kinase (EC 2.7.1.33) & \\
\hline & & $\begin{array}{l}\text { bsu:BSU00710 hs10; } 33 \text { kDa chaperonin; K04083 molecular } \\
\text { chaperone Hsp33 }\end{array}$ & \\
\hline & & bsu:BSU00720 yacD; peptidyl-prolyl cis-trans isomerase & \\
\hline $\mathrm{P}$ & $\mathrm{S}$ & bsu:BSU00730 cysK; cysteine synthase (EC 2.5.1.47) & \\
\hline P & & $\begin{array}{l}\text { bsu: BSU00740 pabB; para-aminobenzoate synthase component I (EC } \\
2.6 .1 .85 \text { ) }\end{array}$ & \\
\hline $\mathrm{P}$ & & $\begin{array}{l}\text { bsu:BSU00750 pabA; para-aminobenzoate/anthranilate synthase } \\
\text { component II (EC } 2.6 .1 .85 \text { ) }\end{array}$ & \\
\hline $\mathrm{P}$ & & bsu:BSU00760 pabc; aminodeoxychorismate lyase (EC 4.1 .3 .38 & \\
\hline $\mathrm{P}$ & & bsu:BSU00770 sul; dihydropteroate synthase (EC 2.5.1.15 & \\
\hline $\mathrm{P}$ & & bsu:BSU00780 folB; dihydroneopterin aldolase (EC 4.1.2.25 & \\
\hline \multirow[t]{3}{*}{$\mathrm{P}$} & & $\begin{array}{l}\text { bsu:BSU00790 folk; 2-amino-4-hydroxy-6-hydroxymethyldihy- } \\
\text { dropteridine pyrophosphokinase }\end{array}$ & \\
\hline & & bsu:BSU00800 yazB; XRE family transcriptional regulator & \\
\hline & & bsu:BSU00810 dusB; tRNA-dihydrouridine synthase (EC 1.-.-.-) & \\
\hline \multirow[t]{3}{*}{$P$} & & bsu:BSU00820 lysS; lysine-tRNA ligase & \\
\hline & & bsu:BSU00830 ctsR; transcriptional regulator CtsR & \\
\hline & & $\begin{array}{l}\text { bsu:BSU00840 mcsA; hypothetical protein; K19411 protein } \\
\text { arginine kinase activator }\end{array}$ & \\
\hline
\end{tabular}

The first line gives the name of the MIBiG cluster containing the considered SM* enzyme, the annotation of the $\mathrm{SM}^{*}$ and the related putative PM enzyme from the same genome, and the BLAST E-value resulting from the comparison of the corresponding two protein sequences

The following lines characterize the \pm 10 genomic neighbourhood of the putative PM enzyme. A "P" in column "E_PM" indicates that this enzyme function, i. e. EC number, occurs in enzymes ${ }_{P_{M}}$ and an "S" in column "K_SM" indicates that KEGG assigned this enzyme function to the pathway "Biosynthesis of secondary metabolites". The column named "KEGG Annotation" lists KEGG-ID, function and EC number of the gene products. The column named "Pathways in $\pm 10 / \pm 2 \mathrm{Nh}$ " lists the number of genes from the corresponding two neighborhoods of the putative PM enzyme that belong to the listed KEGG pathways. For this table, the gene annotations taken from the respective html-page were shortened for the sake of brevity

the annotation given in Table 4 illustrates the difficulties of assigning the function of individual gene products to PM or SM, because 17 entries of the trpE neighborhood are labeled with a "P" and 16 with an "S". Following the link of bsu:BSU22680 (trpE) and clicking the "Genome map" button on the html-page for this KEGG GENES entry, one can easily verify that this neighborhood that contains the genes $\operatorname{trp} \mathrm{A}-\operatorname{trp} \mathrm{E}$ is the trp operon of B. subtilis. Thus, the KEGG PATHWAY annotation "Biosynthesis of secondary metabolites" assigned to 16 gene products of this neighborhood is misleading whereas the less frequently assigned annotation "Phenylalanine, tyrosine and tryptophan biosynthesis" is correct.

The recruitment of trp genes for SM is further documented by the results for BGC0000315, which is the calcium-dependent antibiotic biosynthetic gene cluster from Streptomyces coelicolor (strain ATCC BAA-471/ $\mathrm{A} 3(2) / \mathrm{M} 145)$. The respective html-page shows that this BGC contains the genes $\operatorname{trp} \mathrm{C} 2, \operatorname{tr} p \mathrm{D} 2$, and $\operatorname{trp} \mathrm{E}$, and additional copies of trp genes can be found in the rest of this genome. Our annotation of the SCO7691 neighborhood 
Table 4 Annotation of the \pm 10 genomic neighborhood of trpE from B. subtilis

\begin{tabular}{|c|c|c|c|}
\hline E_PM & K_SM & KEGG Annotation & Pathways in $\pm 10 / \pm 2 \mathrm{Nh}$ \\
\hline & & $\begin{array}{l}\text { bsu:BSU22590 ypiA; TPR repeat-containing protein } \\
\text { YpiA }\end{array}$ & \multirow{21}{*}{$\begin{array}{l}\text { 16/5 bsu01110 Biosynthesis of secondary } \\
\text { metabolites } \\
\text { 13/5 bsu01130 Biosynthesis of antibiotics } \\
\text { 12/5 bsu00400 Phenylalanine, tyrosine and } \\
\text { tryptophan biosynthesis } \\
\text { 12/5 bsu01230 Biosynthesis of amino acids } \\
2 / 0 \text { bsu00401 Novobiocin biosynthesis } \\
2 / 0 \text { bsu00260 Glycine, serine and threonine } \\
\text { metabolism } \\
2 / 0 \text { bsu00900 Terpenoid backbone } \\
\text { biosynthesis } \\
1 / 0 \text { bsu02020 Two-component system } \\
1 / 0 \text { bsu00790 Folate biosynthesis } \\
1 / 0 \text { bsu02030 Bacterial chemotaxis } \\
1 / 0 \text { bsu00240 Pyrimidine metabolism } \\
1 / 0 \text { bsu00230 Purine metabolism } \\
1 / 0 \text { bsu00340 Histidine metabolism } \\
1 / 0 \text { bsu00360 Phenylalanine metabolism } \\
1 / 0 \text { bsu00130 Ubiquinone and other } \\
\text { terpenoid-quinone biosynthesis } \\
1 / 0 \text { bsu00350 Tyrosine metabolism }\end{array}$} \\
\hline $\mathrm{P}$ & $\mathrm{S}$ & $\begin{array}{l}\text { bsu:BSU22600 aroE; } 3 \text {-phosphoshikimate } 1- \\
\text { carboxyvinyltransferase (EC } 2.5 .1 .19 \text { ) }\end{array}$ & \\
\hline$P$ & $\mathrm{~s}$ & $\begin{array}{l}\text { bsu: BSU22610 tyrA; prephenate dehydrogenase (EC } \\
1.3 .1 .12 \text { ) }\end{array}$ & \\
\hline $\mathrm{P}$ & $\mathrm{S}$ & $\begin{array}{l}\text { bsu: BSU2 } 2620 \text { hisC; histidinol-phosphate } \\
\text { aminotransferase (EC } 2.6 .1 .9 \text { ) }\end{array}$ & \\
\hline $\mathrm{P}$ & $\mathrm{S}$ & $\begin{array}{l}\text { bsu:BSU22630 trpA; tryptophan synthase alpha chain } \\
(\text { EC } 4.2 .1 .20)\end{array}$ & \\
\hline $\mathrm{P}$ & $\mathrm{S}$ & $\begin{array}{l}\text { bsu:BSU22640 trpB; tryptophan synthase beta chain } \\
(\text { EC } 4.2 .1 .20)\end{array}$ & \\
\hline$P$ & $\mathrm{~s}$ & $\begin{array}{l}\text { bsu: BSU2 } 2650 \operatorname{trpF} ; \mathrm{N}-\left(5^{\prime}-\right. \\
\text { phosphoribosyl)anthranilate isomerase (EC } \\
5.3 .1 .24)\end{array}$ & \\
\hline $\mathrm{P}$ & $\mathrm{S}$ & $\begin{array}{l}\text { bsu:BSU22660 trpC; indole-3-glycerol phosphate } \\
\text { synthase (EC } 4.1 .1 .48)\end{array}$ & \\
\hline $\mathrm{P}$ & $\mathrm{S}$ & $\begin{array}{l}\text { bsu: BSU2 } 2670 \text { trpD; anthranilate } \\
\text { phosphoribosyltransferase (EC } 2.4 .2 .18 \text { ) }\end{array}$ & \\
\hline$P$ & S & $\begin{array}{l}\text { bsu: BSU22680 trpE; anthranilate synthase component } \\
1 \text { (EC } 4.1 .3 .27)\end{array}$ & \\
\hline $\mathrm{P}$ & $\mathrm{S}$ & $\begin{array}{l}\text { bsu: BSU22690 aroH; chorismate mutase AroH (EC } \\
5.4 .99 .5 \text { ) }\end{array}$ & \\
\hline $\mathrm{P}$ & $\mathrm{S}$ & $\begin{array}{l}\text { bsu: BSU22 } 700 \text { aroB; 3-dehydroquinate synthase (EC } \\
4.2 .3 .4 \text { ) }\end{array}$ & \\
\hline $\mathrm{P}$ & $\mathrm{S}$ & bsu:BSU22710 aroF; chorismate synthase (EC 4.2 .3 .5 ) & \\
\hline $\mathrm{P}$ & & $\begin{array}{l}\text { bsu:BSU } 22720 \text { cheR; chemotaxis protein } \\
\text { methyltransferase (EC } 2.1 .1 .80 \text { ) }\end{array}$ & \\
\hline $\mathrm{P}$ & $\mathrm{S}$ & $\begin{array}{l}\text { bsu:BSU22730 ndk; nucleoside diphosphate kinase (EC } \\
2.7 .4 .6 \text { ) }\end{array}$ & \\
\hline \multirow[t]{2}{*}{$\mathrm{P}$} & $\mathrm{S}$ & $\begin{array}{l}\text { bsu:BSU22 } 740 \text { hepT; heptaprenyl diphosphate synthase } \\
\text { component } 2 \text { (EC } 2.5 .1 .30)\end{array}$ & \\
\hline & $\mathrm{S}$ & $\begin{array}{l}\text { bsu:BSU22750 ubiE; demethylmenaquinone } \\
\text { methyltransferase (EC } 2.1 .1 .- \text { ) }\end{array}$ & \\
\hline \multirow[t]{2}{*}{$\mathrm{P}$} & $\mathrm{S}$ & $\begin{array}{l}\text { bsu:BSU22 } 760 \text { hepS; heptaprenyl diphosphate synthase } \\
\text { component } 1 \text { (EC } 2.5 .1 .30)\end{array}$ & \\
\hline & & $\begin{array}{l}\text { bsu:BSU22770 mtrB; transcription attenuation } \\
\text { protein MtrB }\end{array}$ & \\
\hline \multirow[t]{2}{*}{$\mathrm{P}$} & & $\begin{array}{l}\text { bsu:BSU22780 folE; GTP cyclohydrolase } 1 \text { (EC } \\
3.5 .4 .16 \text { ) }\end{array}$ & \\
\hline & & $\begin{array}{l}\text { bsu:BSU2 } 2590 \text { ypiA; TPR repeat-containing protein } \\
\text { YpiA }\end{array}$ & \\
\hline
\end{tabular}

The first line gives the name of the MIBiG cluster containing the considered $\mathrm{SM}^{*}$ enzyme, the annotation of the $\mathrm{SM}^{*}$ and the related putative PM enzyme from the same genome, and the BLAST E-value resulting from the comparison of the corresponding two protein sequences

The following lines characterize the \pm 10 genomic neighbourhood of the putative PM enzyme. A "P" in column "E_PM" indicates that this enzyme function, i. e. EC number, occurs in enzymes ${ }_{\mathrm{PM}^{*}}$ and an "S" in column "K_SM" indicates that KEGG assigned this enzyme function to the pathway "Biosynthesis of secondary metabolites". The column named "KEGG Annotation" lists KEGG-ID, function and EC number of the gene products. The column named Pathways in $\pm 10 / \pm 2 \mathrm{Nh}$ " lists the number of genes from the corresponding two neighborhoods of the putative PM enzyme that belong to the listed KEGG pathways. For this table, the gene annotations taken from the respective html-page were shortened for the sake of brevity

shown in Table 5 makes clear that this gene does not encode a PM enzyme. Thus, due to the fact that each entry from enzymes species SEGG $_{B C \text { can }}$ possess several homologs in the same genome, the neighborhoods which we supply have to be analyzed carefully by the user in order to assign enzymes to PM or SM.

A further example for a bona fide pair of homologous PM/SM enzymes is shown in Table 6. BGC0000333 is 
Table 5 Annotation of the \pm 10 genomic neighborhood of gene SCO7691 from S. coelicolor

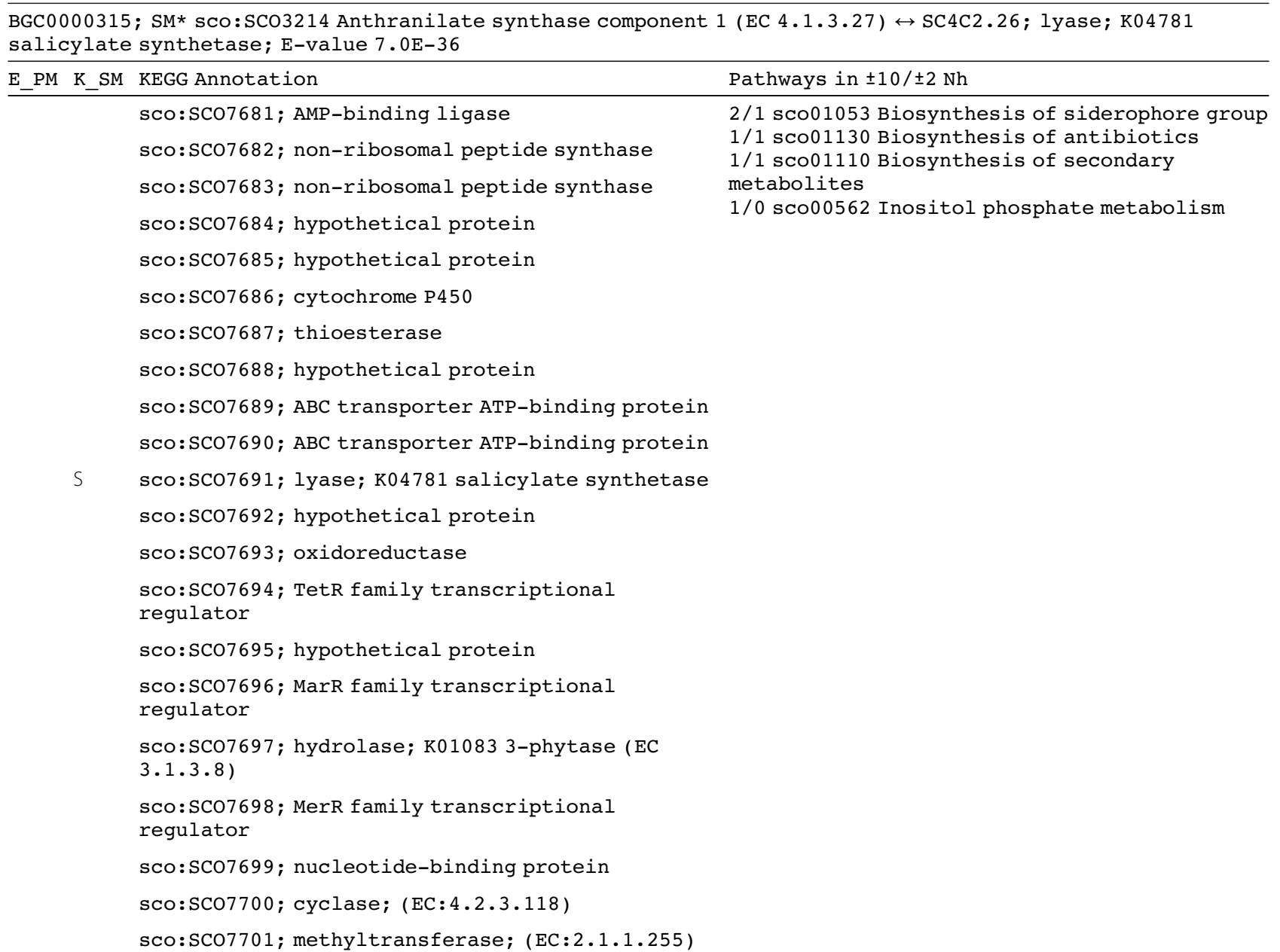

The first line gives the name of the MIBiG cluster containing the considered $\mathrm{SM}^{*}$ enzyme, the annotation of the $\mathrm{SM}^{*}$ and the related putative PM enzyme from the same genome, and the BLAST E-value resulting from the comparison of the corresponding two protein sequences

The following lines characterize the \pm 10 genomic neighbourhood of the putative PM enzyme. A "P" in column "E_PM" indicates that this enzyme function, i. e. EC number, occurs in enzymes ${ }_{P M^{*}}$ and an "S" in column "K_SM" indicates that KEGG assigned this enzyme function to the pathway "Biosynthesis of secondary metabolites". The column named "KEGG Annotation" lists KEGG-ID, function and EC number of the gene products. The column named Pathways in $\pm 10 / \pm 2$ Nh" lists the number of genes from the corresponding two neighborhoods of the putative PM enzyme that belong to the listed KEGG pathways. For this table, the gene annotations taken from the respective html-page were shortened for the sake of brevity

the cyclomarin biosynthetic gene cluster from Salinispora arenicola (strain CNS-205). It contains the gene saq:Sare_4569 that codes for a 4-oxalocrotonate decarboxylase (EC 4.1.1.77). The respective genome contains the two homologs saq:Sare_3899 and saq:Sare_3902 that are involved in benzonate and tryptophan degradation. The extended annotation shown in Table 6 strongly suggests that their genomic neighborhood encodes PM enzymes. Figure 4, which was created by clicking the "Genome map" button of saq:Sare_3902, provides further evidence: The graph confirms that the latter two genes are part of an operon containing enzymes from PM, which illustrates the benefits of using KEGG data.

A compilation of all $B G C_{K E G G}$ html-pages can be downloaded from https://www.bioinf.ur.de. To create this version, we considered all BLAST hits with an Evalue $\leq 1 \mathrm{E}-20$. If one is interested to search homologs of $B G C_{K E G G}$ gene products more sensitively, one only has to follow the links we have integrated. They lead to the respective KEGG GENES entries and one can initiate a BLAST search with a user-defined set of genomes (or a single one) by means of the "DB search" function of KEGG.

\section{Discussion}

The broad functional transition zone that links PM and SM impedes the analysis of SM evolution

Assigning enzyme functions to PM or SM is hampered by several facts. As already mentioned, biosynthetic compounds like lipids or polysaccharides are "essential" for every organism, but the synthesis of some of them in 
Table 6 Annotation of the \pm 10 genomic neighborhood of Sare_3902 from S. arenicola (strain CNS-205)

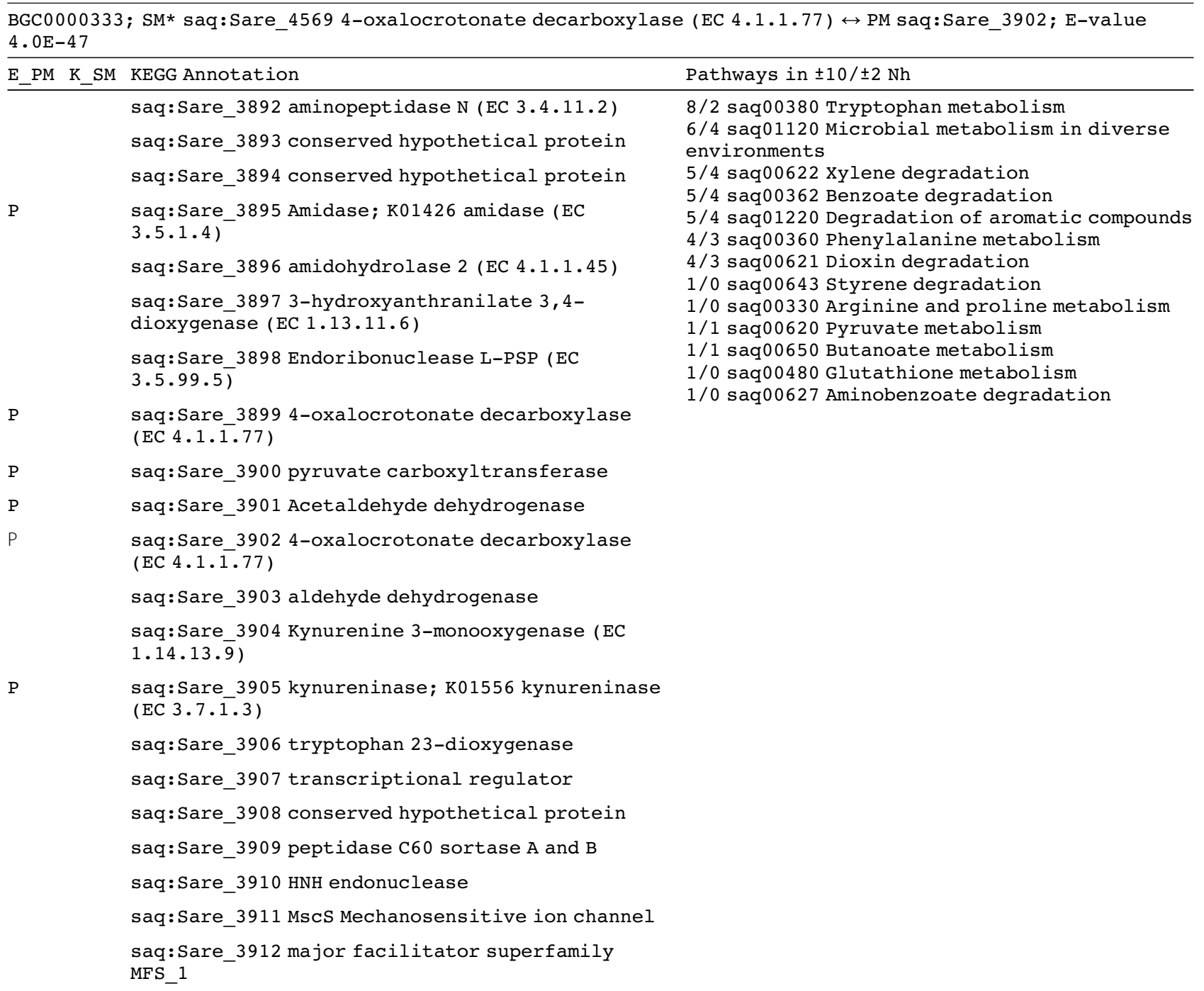

The first line gives the name of the MIBiG cluster containing the considered $\mathrm{SM}^{*}$ enzyme, the annotation of the $\mathrm{SM}^{*}$ and the related putative PM enzyme from the same genome, and the BLAST E-value resulting from the comparison of the corresponding two protein sequences

The following lines characterize the \pm 10 genomic neighbourhood of the putative PM enzyme. A "P" in column " $E \_P M$ " indicates that this enzyme function, i. e. EC number, occurs in enzymes ${ }_{P M^{*}}$ and an "S" in column "K_SM" indicates that KEGG assigned this enzyme function to the pathway "Biosynthesis of secondary metabolites". The column named "KEGG Annotation" lists KEGG-ID, function and EC number of the gene products. The column named "Pathways in $\pm 10 / \pm 2 \mathrm{Nh}$ " lists the number of genes from the corresponding two neighborhoods of the putative PM enzyme that belong to the listed KEGG pathways. For this table, the gene annotations taken from the respective html-page were shortened for the sake of brevity

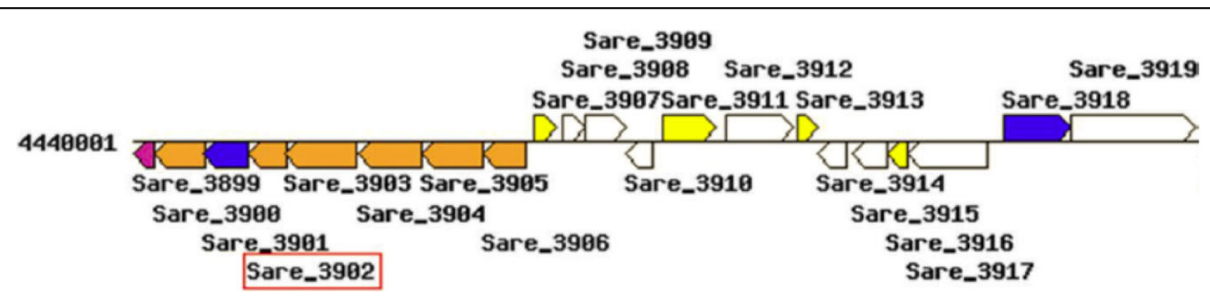

Fig. 4 KEGG genome map for the neighborhood of gene Sare_3902 from S. arenicola. The picture was created by using the "Genome map" function of the KEGG gene entry Sare_3902. KEGG uses the following color code to fill the arrows representing genes: amino acid metabolism (orange), metabolism of cofactors and vitamins (pink), energy metabolism (violet), environmental information processing (yellow), unclassified (white). Sare_3902 codes for a 4-oxalocrotonate decarboxylase; compare Table 6 
SM makes possible a specific interaction of the producing organisms with their environment [9]. Thus, for these enzymatic functions, the assignment to PM or SM is a species-specific problem. Moreover, enzymes like those of the rhamnose biosynthesis pathway supply precursors for PM and SM [32] and the products of tryptophan biosynthesis and other PM pathways are utilized in $\mathrm{SM}$. For these cases, it is difficult to draw the line between PM and SM.

Due to these circumstances, we decided to analyze two subsets, for which PM or SM assignment is highly reliable. However, the surrogates (enzymes ${ }_{P M^{*}}$ and enzymes ${ }_{S M^{*}}$ ) which we compiled, have their specific drawbacks: Most likely, the number of enzymatic functions contributing to bacterial PM and SM is larger than estimated here. As a consequence, the number of SM enzymes recruited from PM is most likely underestimated and the stringent BLAST cutoff [33], which we used to minimize false positives, might additionally contribute to this effect. Thus, we have estimated a lower limit for the functional flexibility of protein folds.

However, despite these limitations, we could deduce several important characteristics of SM enzymes: $i$ ) From the bird's eye view the spectra of enzymatic functions utilized in $\mathrm{SM}^{*}$ and $\mathrm{PM}^{*}$ are highly similar. ii) The finding that $331 \mathrm{SM}^{*}$ functions possess homologous $\mathrm{PM}^{*}$ enzymes strongly support the recruitment theory. iii) Even if we underestimated the functional flexibility of enzymes, we could underpin the broad spectrum of metabolic neofunctionalization, which is exploited by evolution, both in PM and SM.

\section{PM/SM pairs represent a large playground to study enzyme evolution, promiscuity, and their regulatory fine-tuning}

Usually, PM pathways produce single products. For example, the tryptophan biosynthetic pathway makes only tryptophan. In contrast, pathways of SM are diversityoriented and may synthesize up to 100 products [34] which seems puzzling at first glance. However, a decent biological activity is a rare property of a product [9] and thus evolution favors organisms able to generate in SM chemical diversity at low cost. It follows that organisms producing many different compounds improve their fitness, because the number of synthesized products increases the probability that some are biologically active. Along these lines, the wide-spread use of branched and matrix biosynthetic pathways that makes it difficult to distinguish PM and SM enzymes, helps to share metabolic and genetic costs [34].

A further route leading to a widened chemical diversity is the promiscuity of SM enzymes. It has been made plausible that SM enzymes emerged through early gene duplication followed by mutations that broadened substrate selection and flattened activation barriers [35] at the expense of efficiency [20]. Interestingly, it has been shown that promiscuity can be achieved without compromising efficiency [36] and directed evolution and combinatorial engineering approaches are winning strategies to optimize the production of secondary metabolites $[37,38]$. Due to their broader substrate specificity, we propose to consider SM enzymes also for more general enzyme design projects beyond secondary metabolism. Such a strategy has great potential because for at least 391 enzymatic functions that are also relevant in $\mathrm{PM}$, we found at least one enzyme in secondary pathways of bacterial species.

These SM generalists are often slow, because such a catalytic inefficiency is beneficial, $e$. $g$., to avoid competition with primary metabolism [20]. A fine-tuning of enzymatic activities competing for substrates is most critical for homologous PM/SM enzymes that are active in the same cell. In order to identify such cases, we analyzed BGC clusters within their genomic contexts. The resulting species-specific compilation of these $\mathrm{PM} / \mathrm{SM}$ pairs is now an ideal basis for a further in silico analysis and the design of biochemical experiments needed for the detailed characterization of these enzymes and their regulation.

\section{Conclusions}

Secondary, i. e., specialized metabolites produced by bacteria exhibit enormous structural variation and possess a vast range of biological activities. Interestingly, the reaction chemistry used in BGCs to produce these metabolites does not differ drastically from PM. Only few EC subdivisions (i. e. reaction chemistries) are overrepresented in BGCs and for at least 331 enzyme functions found in bacterial BGCs, homologs exist in PM. The functional spectra of homologs are similar, indicating that the evolvability of protein folds is key for establishing novel enzymatic functions, both in PM and SM. Most interesting cases of functional interconversion can be found by scanning the html-pages we provide for each BGC. These homologous PM/SM enzyme pairs are active in the same species and their co-existence may require specific regulatory elements or a fine-tuning of function.

\section{Methods}

\section{Software and databases}

Programs were written in Java (https://java.com/download). Java-based APIs (JAPIs) were used to access the databases BRENDA (SOAP API at http://www.brendaenzymes.org/soap.php), KEGG (REST-API at http:// www.kegg.jp/kegg/rest/keggapi.html), and UniProt (API 
at http://www.ebi.ac.uk/uniprot/remotingAPI). The full genomes of the species listed in Table 1 were assessed by means of KEGG to compile enzymes ${ }_{P M} *$ The MIBiG dataset (version 1.0, http://mibig.secondarymetabolites.org/ repository.html) was downloaded and analyzed locally to deduce $\mathrm{SM}^{*}$ enzymes.

For an unequivocal assignment of function, the above databases were scanned to deduce for each enzyme EC numbers by means of the UniProt ID. Only those enzymes were added to enzymes ${ }_{P M^{*}}$ or enzymes ${ }_{S M^{*}}$, respectively, that had assigned an EC number. To avoid ambiguities, enzymes that were annotated with more than one EC number were eliminated as well; among them were $77 \mathrm{SM}^{*}$ enzymes.

To search for homologs, tblastn and blastp of BLAST [39] were used; for BLASTing KEGG databases, KEGG-BLAST was utilized via the html-page http:// www.genome.jp/tools/blast/. Generally, two enzymes were considered as homologous $(i$. $e$. share the same fold), if the BLAST E-value was $\leq 1 \mathrm{E}-20$. As a control, the cutoff $1 \mathrm{E}-10$ was applied.

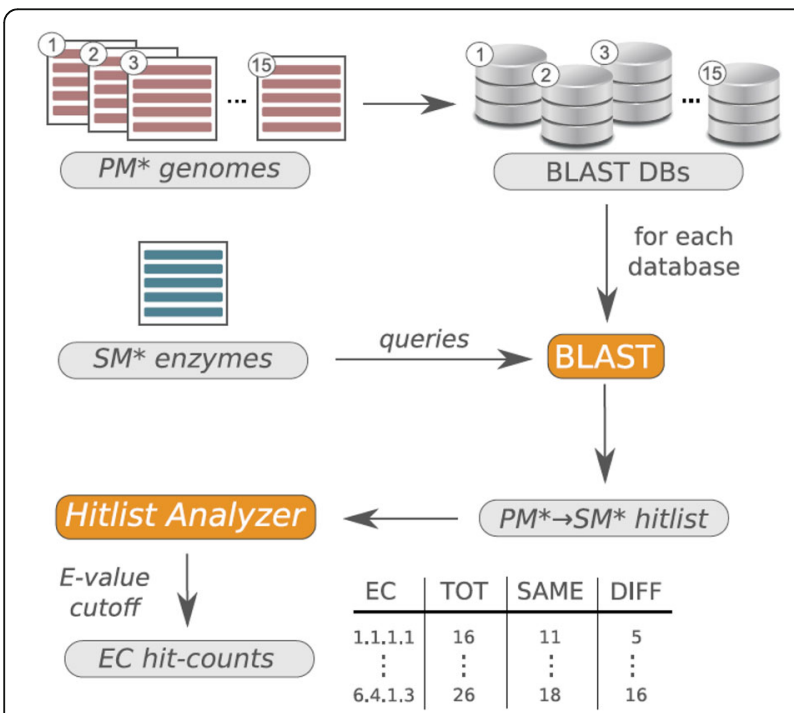

Fig. 5 Software architecture for the determination of $\mathrm{PM}^{*} / \mathrm{SM}^{*}$ homologs. This schema illustrates how the number and the enzyme function of $\mathrm{PM}^{*}$ homologs was determined for $\mathrm{SM}^{*}$ enzymes. For each of the 15 species listed in Table 1, the genome was downloaded from KEGG and the functional assignment was supplemented by using InterPro and other databases. Subsequently, a BLAST database (DB) was built for each of the genomes. The sequences of the $S M^{*}$ enzymes deduced from the chosen BGCs were then BLASTed against all 15 databases. All BLAST hits were stored in $P M^{*} \rightarrow S M^{*}$ hitlist that contained all $\mathrm{PM}^{*} \rightarrow \mathrm{SM}^{*}$ pairs. Based on the chosen E-value cutoff, our program Hitlist Analyzer selected those hits that both had assigned an EC number and compiled the output table EC hits-counts. This table contained for each $E C$ number the number of $\mathrm{PM}^{*}$ hits (TOT) and the number of $\mathrm{PM}^{*}$ hits having assigned the same (SAME) and a different (DIFF) EC number. These raw data were further processed to determine frequencies and related parameters for EC numbers, subdivisions, and classes
To assess the functional variety of enzyme families, BLAST was used to identify for each query $y_{k}$ homologs of all query sequences queries $=\left\{\right.$ query $\left._{k}\right\}$ in a set of reference sequences references $=\left\{\right.$ ref $\left._{l}\right\}$. The XML output of BLAST was parsed and analyzed using Python 2.7 [40] and Biopython [41]. Only those hits $r e f_{l}$ that deviated in length by not more than $30 \%$ from the sequence query were further processed. query $y_{k}$ entries were sorted according to their EC number and the corresponding EC number distribution of their hits was determined and normalized to create the sets $E C_{-} c l$ and $E C_{-} s d$. For a comparison of SM* and $\mathrm{PM}^{*}$ enzymes, the queries were all enzymes ${ }_{S M^{*}}$ sequences and the references were the sequences from enzymes $_{P M^{*}}$. Figure 5 illustrates the software protocol for this case. To determine functional variety in $\mathrm{PM}^{*}$, for each of the 15 query sets enzymes genome $_{i}$, the reference set was references $_{i}=\left\{\right.$ enzymes $_{P M *}$ enzymes $\left._{\text {genome }_{i}}\right\}$. To determine functional variety in $\mathrm{SM}^{*}$, the enzymes of one $B G C_{i}$ constituted the query sets enzymes ${ }_{B G C_{i}}$ and the reference sets were references $i=\left\{\right.$ enzymes $_{S M *}$ enzymes $\left._{B G C_{i}}\right\}$. Additional file 4 contains the sequences of enzymes ${ }_{P M^{*}}$ and Additional file 5 the sequences of enzymes ${ }_{S M}$ *

Relationships between EC classes (EC subdivisions) were visualized as directed graphs using Cytoscape 3.3 and the $\mathrm{yFiles}$ circular layout [28]. Edge widths correspond to the number of the respective BLAST hits; edge colors correspond to the mean E-value of these pairs.

\section{Additional files}

Additional file 1: Table S1. Table in Excel format listing the occurrence of EC numbers, EC subclasses, and of EC subdivisions in enzymes ${ }_{P M^{*}}$ and enzymes $_{S M^{*}}(\mathrm{XLSX} 114 \mathrm{~kb})$

Additional file 2: Table S2. Table in Excel format listing the number of homologous BLAST hits found in enzymes $P_{M^{*}}$ for all enzymes represented in enzymes ${ }_{S *}$. Hits are added according to EC numbers and EC subdivisions. (XLSX 29 kb)

Additional file 3: Figure S1. Figure in PDF format showing the analysis of neofunctionalization based on the BLAST cutoff 1E-10. (PDF $146 \mathrm{~kb}$ )

Additional file 4: 15 multiple Fasta files (1 per genome, compare Table 1) containing the sequences of the data set enzymes PM. The $^{*}$ genomes are named according to KEGG nomenclature. (ZIP 7193 kb)

Additional file 5: 1005 multiple Fasta files (one per BGC that comprises enzymes) containing the sequences of the data set enzymes ${ }_{S M^{*}}$. The BGC are named according to MIBiG nomenclature. (ZIP $6333 \mathrm{~kb}$ )

\section{Acknowledgements}

We thank Michael Bernhard and Wolfgang Kaiser for their help in analyzing the annotation of gene neighborhoods.

\section{Funding}

This work was supported by the Deutsche Forschungsgemeinschaft (award number: ME2259/2-1 to RM, http://www.dfg.de). The funders had no role in study design, data collection and analysis, decision to publish, or preparation of the manuscript.

\section{Availability of data and materials}

The datasets supporting the conclusions of this article are included within the article and its additional files. 


\section{Authors' contributions}

W wrote programs, queried databases and compiled the data. LH performed BLAST searches. MP analyzed the related biochemistry. RM conceived of the project, interpreted the data, and wrote the manuscript. All authors read and approved the final version.

\section{Competing interests}

The authors declare that they have no competing interests.

\section{Author details}

'Institute of Biophysics and Physical Biochemistry, University of Regensburg, D-93040 Regensburg, Germany. ${ }^{2}$ Faculty of Mathematics and Computer Science, University of Hagen, D-58084 Hagen, Germany.

\section{Received: 14 November 2016 Accepted: 16 January 2017}

Published online: 26 January 2017

\section{References}

1. Kossel A. Über die chemische Zusammensetzung der Zelle. Du BoisReymond's Archiv/Arch Anat Physiol Physiol Abt. 1891;8:181-89.

2. Demain AL, Fang A. The natural functions of secondary metabolites. Adv Biochem Eng Biotechnol. 2000;69:1-39.

3. Cong L, Ran FA, Cox D, Lin S, Barretto R, Habib N, Hsu PD, Wu X, Jiang W, Marraffini LA, et al. Multiplex genome engineering using CRISPR/Cas systems. Science. 2013:339(6121):819-23.

4. Leitão AL, Enguita FJ. Secondary metabolism. An unlimited foundation for synthetic biology. Front Microbiol. 2015;6:1562.

5. Medema MH, Kottmann R, Yilmaz P, Cummings M, Biggins JB, Blin K, de Bruijn I, Chooi YH, Claesen J, Coates RC, et al. Minimum information about a biosynthetic gene cluster. Nat Chem Biol. 2015;11(9):625-31.

6. Vining LC. Secondary metabolism, inventive evolution and biochemical diversity - a review. Gene. 1992;115(1-2):135-40.

7. Ziemert N, Lechner A, Wietz M, Millan-Aguinaga N, Chavarria KL, Jensen PR. Diversity and evolution of secondary metabolism in the marine actinomycete genus Salinispora. Proc Natl Acad Sci U S A. 2014;111(12): E1130-1139.

8. Jenke-Kodama H, Muller R, Dittmann E. Evolutionary mechanisms underlying secondary metabolite diversity. Prog Drug Res. 2008;119:121-40.

9. Firn RD, Jones CG. The evolution of secondary metabolism - a unifying model. Mol Microbiol. 2000;37(5):989-94.

10. Dosselaere F, Vanderleyden J. A metabolic node in action: chorismateutilizing enzymes in microorganisms. Crit Rev Microbiol. 2001;27(2):75-131.

11. Plach MG, Löffler P, Merkl R, Sterner R. Conversion of anthranilate synthase into isochorismate synthase: implications for the evolution of chorismateutilizing enzymes. Angew Chem Int Ed. 2015:54(38):11270-4.

12. Jenke-Kodama H, Dittmann E. Evolution of metabolic diversity: insights from microbial polyketide synthases. Phytochemistry. 2009:70(15-16):1858-66.

13. Schomburg I, Chang A, Placzek S, Sohngen C, Rother M, Lang M, Munaretto C, Ulas S, Stelzer M, Grote A, et al. BRENDA in 2013: integrated reactions, kinetic data, enzyme function data, improved disease classification: new options and contents in BRENDA. Nucleic Acids Res. 2013;41(Database issue):D764-772.

14. Kanehisa M, Goto S, Sato Y, Kawashima M, Furumichi M, Tanabe M. Data, information, knowledge and principle: back to metabolism in KEGG. Nucleic Acids Res. 2014;42(1):D199-205.

15. Letzel AC, Pidot SJ, Hertweck C. A genomic approach to the cryptic secondary metabolome of the anaerobic world. Nat Prod Rep. 2013;30(3): 392-428.

16. McDonald AG, Tipton KF. Fifty-five years of enzyme classification: advances and difficulties. FEBS J. 2014:281(2):583-92.

17. IUBMB Enzyme Nomenclature [http://www.chem.qmul.ac.uk/iubmb/ enzyme/]. Accessed Oct 2016

18. Martinez Cuesta S, Furnham N, Rahman SA, Sillitoe I, Thornton JM. The evolution of enzyme function in the isomerases. Curr Opin Struct Biol. 2014; 26:121-30.

19. Stothard P, Van Domselaar G, Shrivastava S, Guo A, O'Neill B, Cruz J, Ellison M, Wishart DS. BacMap: an interactive picture atlas of annotated bacterial genomes. Nucleic Acids Res. 2005;33(Database issue):D317-320.

20. Bar-Even A, Tawfik DS. Engineering specialized metabolic pathways-is there a room for enzyme improvements? Curr Opin Biotechnol. 2013;24(2):310-9.
21. Banerjee R, Becker D, Dickman M, Gladyshev V, Ragsdale S. Redox biochemistry. Hoboken: Wiley-Interscience; 2008.

22. Cimermancic P, Medema MH, Claesen J, Kurita K, Wieland Brown LC, Mavrommatis K, Pati A, Godfrey PA, Koehrsen M, Clardy J, et al. Insights into secondary metabolism from a global analysis of prokaryotic biosynthetic gene clusters. Cell. 2014;158(2):412-21.

23. Rost B. Twilight zone of protein sequence alignments. Protein Eng. 1999; 12(2):85-94.

24. Pearson WR. An introduction to sequence similarity ("homology") searching Curr Protoc Bioinformatics. 2013;42:3.1.1-3.1.8.

25. Rost B. Enzyme function less conserved than anticipated. J Mol Biol. 2002 318(2):595-608.

26. Furnham N, Sillitoe I, Holliday GL, Cuff AL, Laskowski RA, Orengo CA, Thornton JM. Exploring the evolution of novel enzyme functions within structurally defined protein superfamilies. PLoS Comp Biol. 2012:8(3): e1002403.

27. Bartlett GJ, Borkakoti N, Thornton JM. Catalysing new reactions during evolution: economy of residues and mechanism. J Mol Biol. 2003;331(4): 829-60.

28. Smoot ME, Ono K, Ruscheinski J, Wang PL, Ideker T. Cytoscape 2.8: new features for data integration and network visualization. Bioinformatics. 2011; 27(3):431-2.

29. Blake JA, Harris MA. The Gene Ontology (GO) project: structured vocabularies for molecular biology and their application to genome and expression analysis. Curr Protoc Bioinformatics. 2008;23:7.2.1-7.2.9.

30. Price MN, Arkin AP, Alm EJ. The life-cycle of operons. PLoS Genet. 2006: 2(6):e96.

31. Zheng Y, Szustakowski JD, Fortnow L, Roberts RJ, Kasif S. Computational identification of operons in microbial genomes. Genome Res. 2002;12(8): 1221-30.

32. Madduri K, Waldron C, Merlo DJ. Rhamnose biosynthesis pathway supplies precursors for primary and secondary metabolism in Saccharopolyspora spinosa. J Bacteriol. 2001:183(19):5632-8.

33. Deluca TF, Wu IH, Pu J, Monaghan T, Peshkin L, Singh S, Wall DP. Roundup: a multi-genome repository of orthologs and evolutionary distances. Bioinformatics. 2006;22(16):2044-6.

34. Fischbach MA, Clardy J. One pathway, many products. Nat Chem Biol. 2007; 3(7):353-5.

35. Weng JK, Philippe RN, Noel JP. The rise of chemodiversity in plants. Science. 2012:336(6089):1667-70.

36. Copley SD. An evolutionary biochemist's perspective on promiscuity. Trends Biochem Sci. 2015:40(2):72-8

37. Lee PC, Momen AZ, Mijts BN, Schmidt-Dannert C. Biosynthesis of structurally novel carotenoids in Escherichia coli. Chem Biol. 2003:10(5):453-62.

38. Mijts BN, Schmidt-Dannert C. Engineering of secondary metabolite pathways. Curr Opin Biotechnol. 2003;14(6):597-602.

39. Altschul SF, Madden TL, Schaffer AA, Zhang J, Zhang Z, Miller W, Lipman DJ. Gapped BLAST and PSI-BLAST: a new generation of protein database search programs. Nucleic Acids Res. 1997;25(17):3389-402.

40. Python Software Foundation. Python Language Reference, version 2.7 [http://www.python.org]

41. Cock PJ, Antao T, Chang JT, Chapman BA, Cox CJ, Dalke A, Friedberg I, Hamelryck T, Kauff F, Wilczynski B, et al. Biopython: freely available python tools for computational molecular biology and bioinformatics. Bioinformatics. 2009:25(11):1422-3. 